\title{
Clinicopathological study of hepatocellular carcinoma with peliotic change
}

\author{
MASARU FUJIMOTO ${ }^{1,2}$, OSAMU NAKASHIMA ${ }^{1}$, MINA KOMUTA ${ }^{1}$, \\ TOSHIMITSU MIYAAKI ${ }^{1}$, MASAMICHI KOJIRO $^{1}$ and HIROHISA YANO ${ }^{1}$
}

\author{
${ }^{1}$ Department of Pathology, Kurume University School of Medicine, Kurume, Fukuoka 830-0011; \\ ${ }^{2}$ Division of Hepatology and Metabolism, Department of Internal Medicine, Faculty of Medicine, \\ Saga University, Nabeshima 849-8501, Japan
}

Received May 25, 2009; Accepted September 23, 2009

DOI: 10.3892/ol_00000003

\begin{abstract}
Peliosis hepatis-like blood-filled cavities are frequently observed in the tumors of hepatocellular carcinoma (HCC). This finding is generally referred to as 'peliotic change' in HCC. However, the clinicopathological features of HCC with peliotic change (PHCC) are not fully understood. These issues are addressed in the present study. Among 294 consecutively surgically resected HCCs, the clinicopathological features of PHCC were compared with those of a common type of HCC (control). PHCC was observed in $116(39.5 \%)$ of 294 HCCs. The mean tumor diameter of $3.4 \pm 0.9 \mathrm{~cm}$ of the PHCC group was significantly larger than that of the $2.5 \pm 0.9 \mathrm{~cm}$ of the control, and the incidence of PHCC was related to increased tumor diameter. In the 116 PHCCs, the tumors were completely or incompletely encapsulated. On ultrasonography, PHCCs showed hyperechoic and/or mosaic patterns. The mean diameter of $3.5 \pm 0.8 \mathrm{~cm}$ of PHCCs with a hyperechoic and/or mosaic pattern was significantly larger than that of $2.3 \pm 0.9 \mathrm{~cm}$ in the control. In conclusion, it is necessary for clinicians and pathologists to discern the characteristics of peliotic change as a morphological feature that modifies ultrasound findings.
\end{abstract}

\section{Introduction}

Peliosis hepatis, a hepatic lesion characterized by blood-filled parenchymal cavities randomly scattered throughout the liver (1-3), was first described by Wagner (4), but its pathogenesis is a matter of debate. Peliosis hepatis-like blood-filled cavities are also frequently observed in the tumors of hepatocellular carcinoma (HCC) (5-7). This finding is generally referred to

Correspondence to: Dr Hirohisa Yano, Department of Pathology, Kurume University School of Medicine, 67 Asahi-machi, Kurume, Fukuoka 830-0011, Japan

E-mail: hiroyano@med.kurume-u.ac.jp

Key words: hepatocellular carcinoma, peliotic change, peliosis hepatis, ultrasound, mosaic pattern as 'peliotic change' in HCC. Almost no clinicopathological assessment of this peliotic change has been conducted, and it is still considered little more than an often observed incidental or accidental finding. However, along with the advances in diagnostic imaging, peliotic change has drawn attention as a morphological feature that modifies image findings of HCC.

In the present study, we conducted a clinicopathological study of HCC with peliotic change (PHCC).

\section{Materials and methods}

A total of 294 HCCs without preoperative anticancer therapies were consecutively resected at Kurume University Hospital between January 1991 and December 2003. Cases showing a peliosis hepatis-like change (peliotic change) in the tumor were included for the study as PHCC and compared with cases of a common type of HCC as control. The resected liver specimens were fixed in $10 \%$ buffered formalin immediately after hepatectomy, cut serially into $5 \mathrm{~mm}$ slices and macroscopically examined. Sections containing tumor tissues as well as the surrounding liver tissues were embedded in paraffin, cut into $4-\mu \mathrm{m}$ sections and routinely stained with hematoxylin and eosin. Immunohistochemical staining of CD34 was performed on 20 PHCC cases to examine the endothelial cells of the sinusoidal blood spaces of the tumor, using mouse monoclonal antibody against CD34 (anti-CD34; Dako, CA, USA) and the Streptavidin Peroxidase technique (MaxiTags kits, Immunon $^{\mathrm{TM}}$, Lipshaw, PA, USA). Clinical data were obtained from clinical charts. Informed consent was obtained from the patients included in the study.

Statistical analysis was performed using Stat View version J-5.0 (Abacus Concepts Inc., Berkeley, CA, USA). Difference of means was assessed by the unpaired Student's t-test or Mann-Whitney $\mathrm{U}$ test. $\mathrm{P}<0.05$ was considered statistically significant.

\section{Results}

Clinical findings of PHCC. PHCC was observed in 116 (39.5\%) out of 294 cases. Ages ranged from 41 to 78 years (mean 63.2 \pm 7.8 SD) in the PHCCs and from 16 to 80 years (mean 64.5 $\pm 8.8 \mathrm{SD}$ ) in the control group. The PHCC group 
Table I. Comparison of the ultrasonographic pattern between hepatocellular carcinoma with peliotic change and a common type of HCC (control) according to the tumor size.

\begin{tabular}{|c|c|c|c|c|c|c|}
\hline Tumor size $(\mathrm{cm})$ & $0.0-1.0$ & $1.1-2.0$ & $2.1-3.0$ & $3.1-4.0$ & $4.1-5.0$ & Total $(\%)$ \\
\hline \multicolumn{7}{|l|}{ PHCC group } \\
\hline Hyperechoic & 0 & 0 & 3 & 5 & 4 & $12(20)$ \\
\hline Mosaic & 0 & 1 & 7 & 5 & 5 & $18(31)^{\mathrm{a}}$ \\
\hline Isoechoic & 0 & 2 & 2 & 2 & 2 & 8 (14) \\
\hline Hypoechoic & 0 & 4 & 7 & 10 & 0 & 21 \\
\hline Total & 0 & 7 & 19 & 22 & 11 & $59(100)$ \\
\hline \multicolumn{7}{|l|}{ Control group } \\
\hline Hyperechoic & 3 & 7 & 6 & 1 & 0 & 17 (19) \\
\hline Mosaic & 0 & 3 & 6 & 1 & 1 & 11 (13) \\
\hline Isoechoic & 2 & 3 & 6 & 5 & 2 & $18 \quad(20)$ \\
\hline Hypoechoic & 2 & 17 & 19 & 4 & 0 & 42 (48) \\
\hline Total & 7 & 30 & 37 & 11 & 3 & $88(100)$ \\
\hline
\end{tabular}

${ }^{\mathrm{a}}<0.01$ vs. mosaic in control.

included 89 males and 27 females (3.3:1), while the control group comprised 140 males and 38 females (3.7:1). No significant difference was noted in gender between the two groups.

Hepatitis B surface antigen ( $\mathrm{HBsAg}$ ) was found to be positive in 16 cases $(15 \%)$ out of 104 in the PHCC group and in 20 cases $(12 \%)$ out of 163 in the control group. Hepatitis C virus antibody (HCVAb) was found to be positive in 82 cases (77\%) out of 107 in the PHCC group and in 132 cases (80\%) out of 166 cases in the control group, indicating no significant difference between the two groups. In the remaining 27 and 21 of the 294 cases, respectively, HBsAg- and HCVAb-positives were unknown.

The laboratory data for asparate aminotransferase, alanine aminotransferase, albumin, as well as platelet and serum $\alpha$-fetoprotein were not significantly different between the two groups.

Imaging findings of PHCC. Among 59 PHCCs in which abdominal ultrasound findings were available, 12 (20\%) had a hyperechoic pattern and $18(31 \%)$ a mosaic pattern. Among 88 cases of the control group, 17 (19\%) had a hyperechoic pattern and $11(13 \%)$ a mosaic pattern, indicating significantly more lesions with a mosaic pattern in the PHCC group $(\mathrm{P}<0.01$; Table I). Furthermore, the mean tumor diameter in the cases with a hyperechoic pattern and/or mosaic pattern was $3.5 \pm 0.8 \mathrm{~cm}$ in the PHCC group and $2.3 \pm 0.9 \mathrm{~cm}$ in 28 controls. The tumor size of PHCCs with hyperechoic and/or mosaic patterns was significantly larger than that of the control $(\mathrm{P}<0.001)$.

In the majority of 50 cases in the PHCC group that underwent dynamic CT scans, typical HCC patterns were observed, such as high attenuation in the early enhanced phase and wash-out in the delay enhanced phase. No specific difference in CT findings was noted in PHCCs.

Pathological findings of PHCC. Tumor diameter ranged from 1.5 to $5.0 \mathrm{~cm}$ (average $3.4 \pm 0.9 \mathrm{SD}$ ) in the PHCC group and
Table II. Comparison in tumor size between hepatocellular carcinoma with peliotic change and a common type of HCC (control).

\begin{tabular}{lrrr}
\hline $\begin{array}{l}\text { Tumor size }(\mathrm{cm}) \\
\text { Average } \pm \text { SD }\end{array}$ & $\begin{array}{r}\text { PHCC }(\%) \\
3.4 \pm 0.9^{\mathrm{a}}\end{array}$ & $\begin{array}{c}\text { Control }(\%) \\
2.5 \pm 0.9\end{array}$ \\
\hline $0.0-1.0$ & 0 & $(0)$ & $11(100)$ \\
$1.1-2.0$ & 10 & $(16)$ & $54 \quad(84)$ \\
$2.1-3.0$ & 37 & $(32)$ & $77(68)$ \\
$3.1-4.0$ & 44 & $(61)$ & $28(39)$ \\
$4.1-5.0$ & $25(76)$ & $8 \quad(24)$ \\
Total & $116(100)$ & $178(100)$ \\
\hline
\end{tabular}

aP $<0.001$ vs. control.

from 0.7 to $4.8 \mathrm{~cm}$ (average $2.5 \pm 0.9 \mathrm{SD}$ ) in the control group, indicating significantly larger tumors in the PHCC group $(\mathrm{P}<0.001$; Table II). The incidence of PHCC was related to the increase of tumor diameter. Tumors $<2 \mathrm{~cm}$ were found in 10 cases (9\%) in the PHCC group and in 65 cases (37\%) in the control. No tumors $<1 \mathrm{~cm}$ in diameter were detected in the PHCC group.

The tumors were completely encapsulated in 108 (93\%) of the 116 PHCCs, but incompletely encapsulated in the remaining 8 cases. On the other hand, encapsulated HCC was observed in $106(60 \%)$ out of 178 tumors in the control group, indicating a significantly higher frequency of encapsulation in the PHCC group $(\mathrm{P}<0.001)$. Peliotic change was observed as varying sized blood lakes and hemorrhagic honeycomb-like appearance (Fig. 1A and B). In some cases, peliotic changes occupied $\sim 2 / 3$ of the cut surface of the tumor. Peliotic changes were easily distinguished from hemorrhage because the latter was accompanied by degeneration and/or necrosis of the tumor 


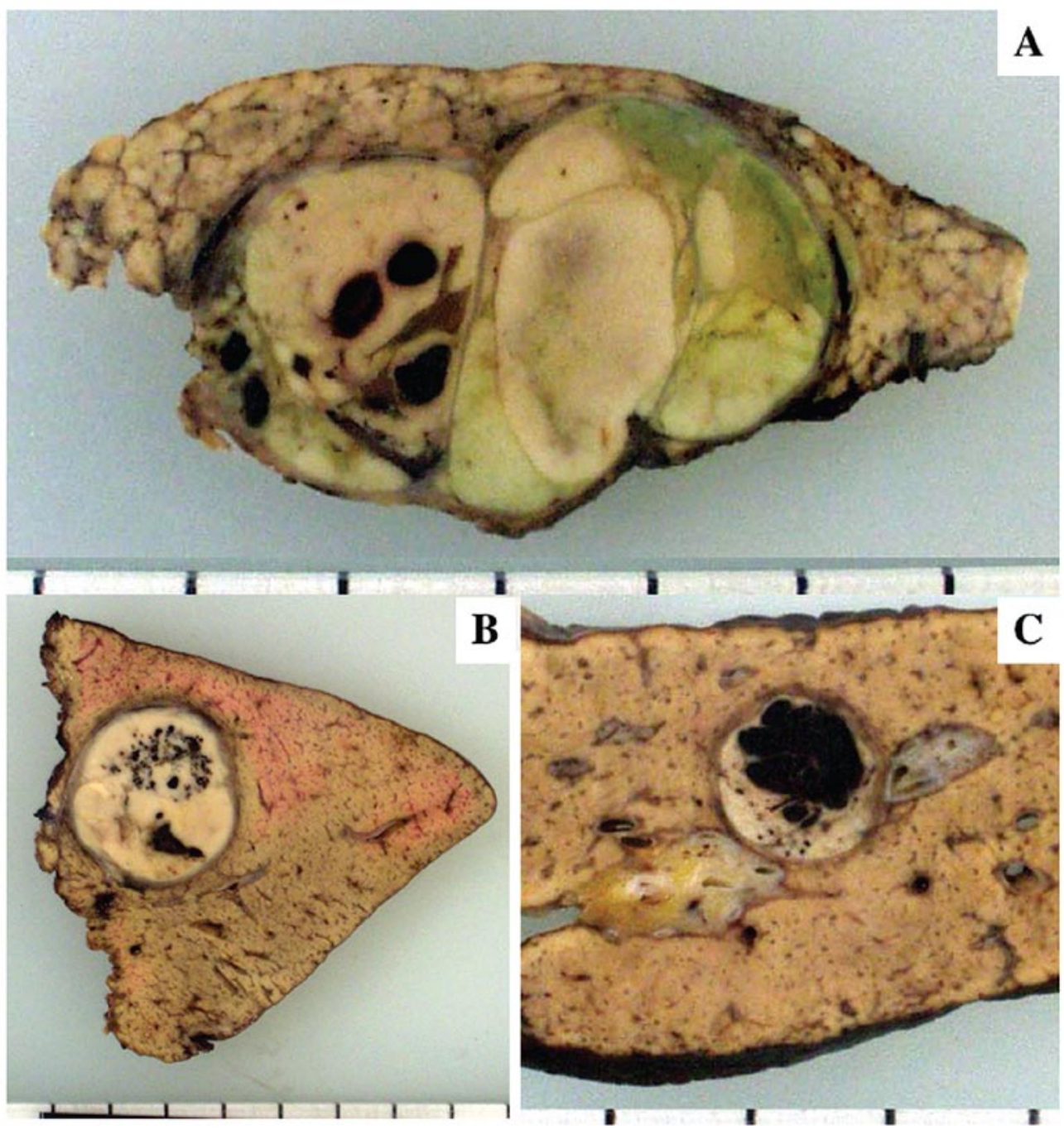

Figure 1. Macroscopic features of HCC with peliotic changes. (A) Dot-like peliotic changes. (B) Honeycomb-shaped and small blood lake-like peliotic changes in encapsulated tumor. (C) Blood lake-like peliotic change with clear boundaries occupying $\sim 2 / 3$ of the cut surface of the tumor.
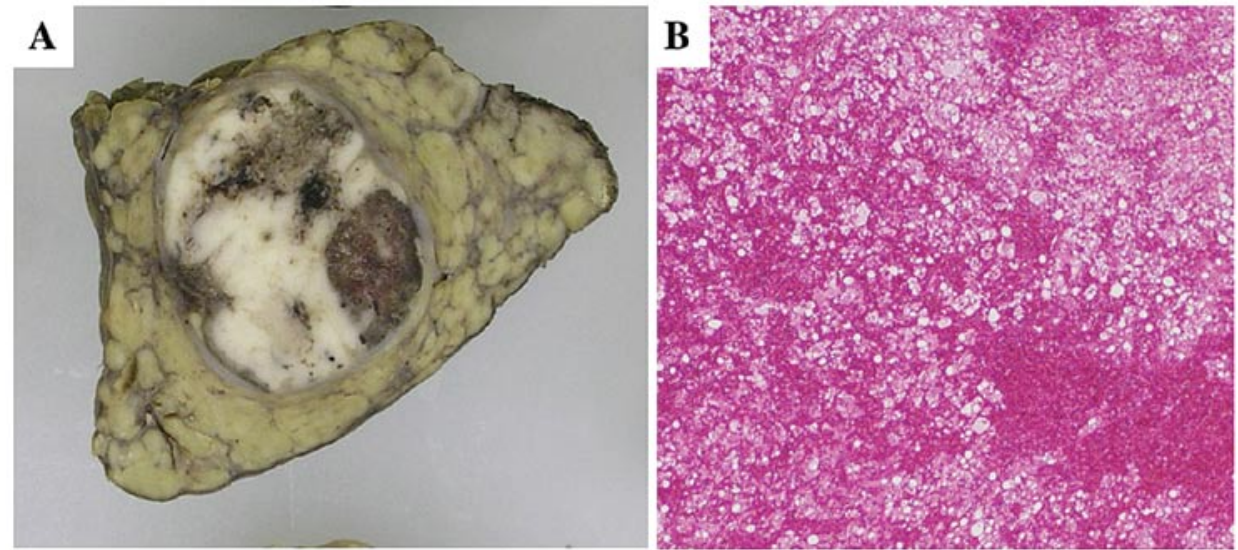

Figure 2. Hemorrhage in HCC. (A) Hemorrhage is observed in the degenerative and necrotic areas of the tumor. (B) Histologically diffuse hemorrhage is observed in degenerative HCC tissue.

tissue (Fig. 2). Fibrous septa were observed in 76 PHCCs (68\%) and in 112 cases (63\%) in the control group.

Peliotic change was observed as varying sized blood lakes without obvious lining of the endothelial cells (Fig. 3A). The lack of endothelial cells was also confirmed by immunostaining for CD34 (Fig. 3B). The majority of PHCCs were moderately differentiated showing a trabecular or pseudoglandular or both patterns. No well-differentiated type was found. Degeneration 

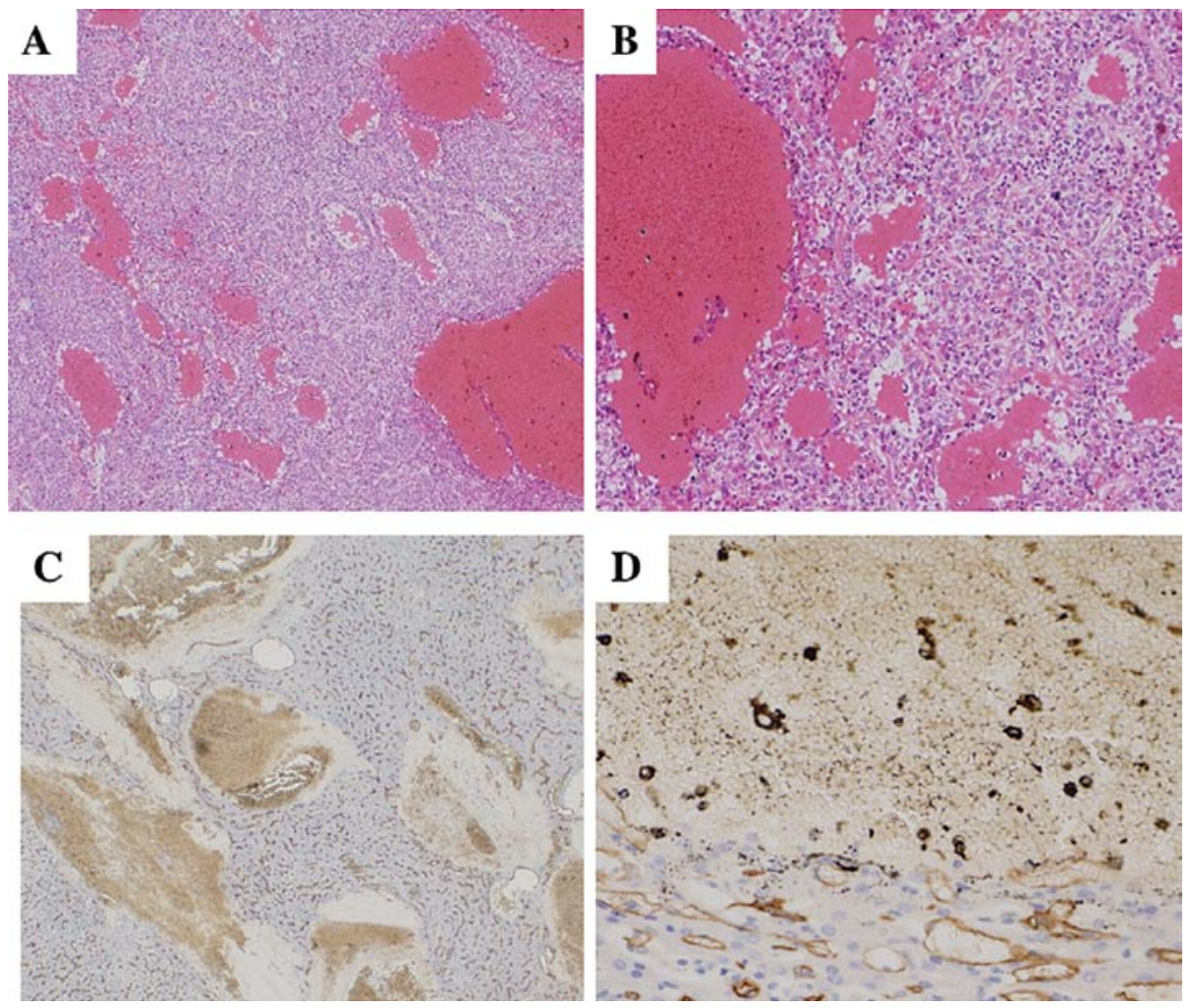

Figure 3. Histological features of peliotic changes. (A and B) Small and large blood lakes are observed in moderately differentiated HCC and irregular dilatation of sinusoid-like blood spaces of the tumor (H\&E stain). (C and D) Immunostaining for CD34 shows no positive cells along the spaces of peliotic change.

or necrosis was not observed around peliotic changes. A varying degree of sinusoidal dilatation was observed around peliotic changes. In the control group, 116 tumors $(66 \%)$ were moderately differentiated, 55 tumors $(31 \%)$ were poorly or moderately to poorly differentiated and 6 tumors $(3 \%)$ were well-differentiated. Among 111 PHCCs in which noncancerous areas were examined, 66 cases (59\%) were chronic hepatitis associated with varying degrees of fibrosis and 45 (41\%) were liver cirrhosis. Among the 176 cases in the control group, 85 cases (48\%) were chronic hepatitis and 91 (52\%) cirrhosis. No significant difference was ntoed in the background liver between the groups.

Recurrence rate after resection. Follow-up data following surgery were obtained in 32 PHCC cases and in 39 controls. Follow-up periods ranged from 4 to 105 months (mean $38 \pm 25.7$ SD). Recurrence occurred in 18 PHCC cases (56\%) and in 17 controls (44\%). There was no significant difference between the 2 groups.

\section{Discussion}

Peliotic changes are frequently observed in HCCs. However, the question raised is whether peliotic change is essentially different from hemorrhage of the tumor. In general, degenerative change and/or necrosis are observed around hemorrhage of HCC tumors. Put differently, degeneration and/or necrosis of tumor tissue cause hemorrhage in HCC. On the other hand, tumor tissues around peliotic changes are not degenerative or necrotic and blood is localized within the spaces. Thus, it is suggested that peliotic changes are different from hemorrhage.
However, when peliotic changes become extensive, they may rupture and hemorrhage may develop.

Various factors are suggested in the pathogenesis of peliosis hepatis. These factors are excessive alcohol intake, hormonal agents such as oral contraceptives and anabolic steroids, as well as chronic wasting diseases, including malignant tumors and tuberculosis (8-12). It was also suggested that blood constituents infiltrate the Disse's spaces resulting in cyst-like change of the sinusoids following the mechanical disorder of the sinusoidal endothelial cells (13). In a study of 12 cases of peliosis hepatis, Zafrani et al suggested that the developmental mechanism of peliosis hepatis obstructed blood channels caused by sinusoidal destruction accompanying hepatocellular necrosis or the abnormal bonding of sinusoids and the central vein (2). Based on the fact that peliotic changes were frequently observed in the encapsulated tumors, the mechanism of peliotic change in HCC was explained by endothelial damage due to sinusoidal dilatation following increased intratumoral pressure. This assumption is supported by the fact that similar peliotic changes are frequently observed in liver cell adenoma, which is also an expansive tumor with frequent encapsulation. Sugimachi et al suggested a particular relationship between peliotic change in HCC and angiopoietin-2 (Ang-2), which play a regulatory role in tumor vessel remodeling (14).

No significant clinical differences were noted between the PHCC and control groups other than the significantly higher frequency of hyperechoic and/or mosaic patterns in PHCCs on ultrasonography. The most common histological feature reflecting hyperechogenecity in HCC is fatty change of the tumor which most frequently occurs in small well-differentiated tumors up to $\sim 2 \mathrm{~cm}$ in diameter (15). HCC tumors with 
hyperechoic and/or mosaic patterns among tumors $>3-4 \mathrm{~cm}$ in diameter are rarely found. In the present study, these tumors proved to be PHCCs.

Although peliotic change in HCC has little clinical significance, it is necessary for clinicians and pathologists to distinguish the presence of peliotic change as a morphological feature that modifies ultrasonographic patterns of HCC.

\section{Acknowledgements}

We thank Ms. Sachiyo Maeda and Ms. Misato Shiraishi for their technical assistance on immunohistochemical staining. This study was supported in part by the Sarah Cousins Memorial Fund, Boston, MA, USA.

\section{References}

1. Karasawa T, Shikata T and Roger DS: Peliosis hepatis: report of nine cases. Acta Pathol Jpn 29: 457-469, 1979.

2. Zafrani ES, Cazier A, Baudelot AM and Feldmann G: Ultrastructural lesions of the liver in human peliosis. A report of 12 cases. Am J Pathol 114: 349-359, 1984.

3. Asano S, Wakasa H, Kaise S, Nishimaki T and Kasukawa R: Peliosis hepatis: Report of two autopsy cases with a review of literature. Acta Pathol Jpn 32: 861-877, 1982.

4. Wagner E: Fall von Blutcysten der Leber (In German). Arch Heilk 2: 369-370, 1861

5. Kojiro M: Peliosis hepatis (In Japanese). Acta Hepatol Jpn (KANZO) 38: 583-586, 1997.
6. Grazioli L, Morana G, Caudana R, et al: Hepatocellular carcinoma: correlation between gadobenate dimeglumine-enhanced MRI and pathological findings. Invest Radiol 35: 25-34, 2000.

7. Brancatelli G, Baron RL, Peterson MS and Marsh W: Helical CT screening for hepatocellular carcinoma in patient with cirrhosis: frequency and cases of false-positive interpretation. Am J Roentgenol 180: 1007-1014, 2003.

8. Loomus GN, Aneja P and Bota RA: A case of peliosis hepatis in association with tamoxifen therapy. Am J Clin Pathol 80: 881-882, 1983.

9. Dejgaard A, Krogsgaard K and Jacobsen M: Veno-occlusive disease and peliosis of the liver after thorotrast administration. Virchows Arch A Pathol Anat Histopathol 403: 87-94, 1984.

10. Czapar CA, Weldon-Linne CM, Moore DM and Rhone DP: Peliosis hepatis in the acquired immunodeficiency syndrome. Arch Pathol Lab Med 110: 611-613, 1986.

11. Russmann S, Zimmermann A, Krahenbuhl S, Kern B and Reichen J: Veno-occlusive disease, nodular regenerative hyperplasia and hepatocellular carcinoma after azathioprine treatment in a patient with uncreative colitis. Eur J Gastroenterol Hepatol 13: 287-290, 2001.

12. Drevelengas A, Chourmouzi D and Boulogianni G: Peliosis of the liver in a patient with prostate carcinoma. JBR-BTR 86: 158-159, 2003.

13. Shim SG, Paik SW, Hyun JG, et al: Lipiodol accumulation in focal peliosis hepatis with sinusoidal dilatation. J Clin Gastroenterol 32: 356-358, 2001.

14. Sugimachi K, Tanaka S, Taguchi K, Aishima S, Shimada M and Tsuneyoshi M: Angiopoietin switching regulates angiogenesis and progression of human hepatocellular carcinoma. J Clin Pathol 56: 854-860, 2003.

15. Kutami R, Nakashima Y, Nakashima O, Shiota K and Kojiro M: Pathomorphologic study on the mechanism of fatty change in small hepatocellular carcinoma of humans. J Hepatol 33: 282-289, 2000. 